\title{
Gradhiva
}

GRADHI

Revue d'anthropologie et d'histoire des arts

29 | 2019

Estrangemental

\section{Cosmogonie et cosmologie}

Philip K. Dick

Traducteur : Hélène Collon

\section{CpenEdition}

\section{Journals}

Édition électronique

URL : http://journals.openedition.org/gradhiva/4000

DOI : $10.4000 /$ gradhiva. 4000

ISSN : 1760-849X

Éditeur

Musée du quai Branly Jacques Chirac

Édition imprimée

Date de publication : 29 mai 2019

Pagination : $30-49$

ISBN : 978-2-35744-112-5

ISSN : 0764-8928

\section{Référence électronique}

Philip K. Dick, «Cosmogonie et cosmologie », Gradhiva [En ligne], 29 | 2019, mis en ligne le 31 mars

2021, consulté le 02 avril 2021. URL : http://journals.openedition.org/gradhiva/4000 ; DOI : https:// doi.org/10.4000/gradhiva.4000

(C) musée du quai Branly 


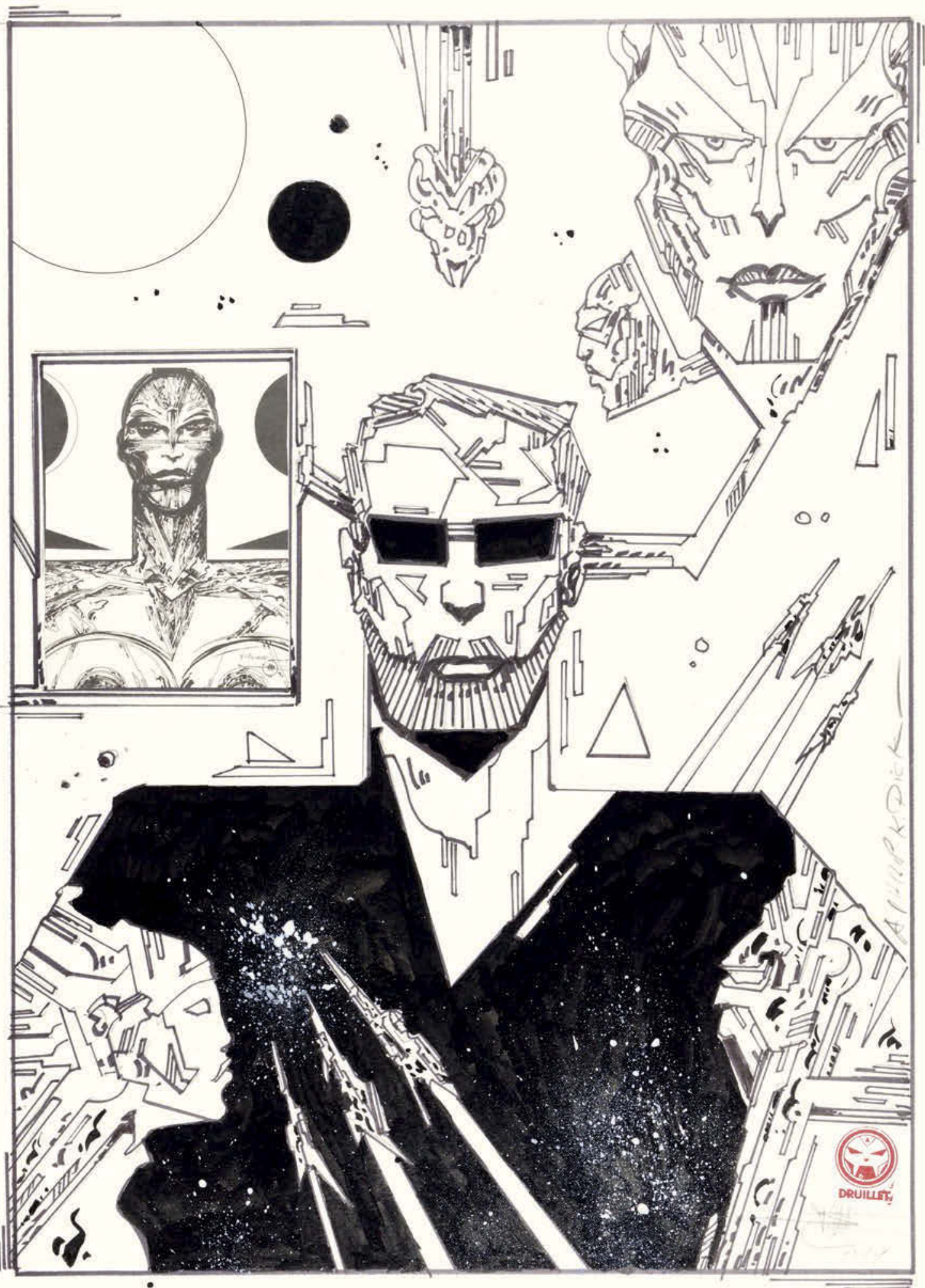




\section{Cosmogonie et cosmologie* Par Philip K. Dick}

* Titre original:

Cosmogony and

Cosmology @ 1987

The Estate of Philip K. Dick

All rights reserved.

Inédit en français, "Cosmologie et cosmogonie» est un étrange essai spéculatif de Dick représentatif de sa seconde période (dite «mystique»). Comment expliquer l'anomalie, l'imperfection ou l'incomplétude fondamentale de notre monde? En quoi un sandwich au jambon fait-il un bon modèle de réalité ? Et si la théologie était au fond de la sciencefiction et vice-versa? Voici quelques-unes des questions posées par cet exercice de cosmologie expérimentale où théologie et science-fiction deviennent une seule et même chose: un exercice spéculatif d'invention d'hypothèses de grande ampleur sur le travail des entités à l'œuvre au sein du cosmos, et au terme duquel la réalité se trouve plus ou moins acceptée, niée, modifiée ou réévaluée dans son statut d'apparence phénoménale.

L'écrivain puise ici à des sources d'inspirations diverses, gnostiques, chrétiennes et orientales pour se fabriquer une croyance viable mariant théologie et technologie. II reprend notamment au théosophe allemand Jakob Böhme (1575-1624) I'hypothèse que Dieu serait en quelque sorte au stade larvaire, naîtrait à la conscience et aurait produit une image (notre monde) comme un grand exercice d'introspection pour se connaître lui-même, via un appareil de projection plus ou moins défaillant (Zebra). Dick se déclare plus loin «panenthéiste», notion qu'il emprunte au philosophe allemand Karl Christian Friedrich Krause (1781-1832). Tandis que le panthéisme affirme que tout est divin et que la divinité possède les mêmes limites spatio-temporelles que le cosmos, le panenthéisme postule l'existence d'une Entité bien plus grande que l'univers dont celui-ci n'est finalement qu'une partie. L'écrivain de science-fiction se range donc du côté d'Aristote, de Plotin, Maître Eckhart, Spinoza, Whitehead, de l'advaita vedanta, auxquels on aurait pu ajouter les prêtres aztèques aussi bien que beaucoup de peuples d'Amérique du nord. II se déclare par ailleurs «acosmique» (expression empruntée à la philosophie de la religion de Hegel), voyant dans l'aspect phénoménal du cosmos soit une illusion d'optique soit une réalité incomplète ou inachevée, une idée partagée par Shankara, Platon, Spinoza, Hegel, Schopenhauer ou encore Whitehead. 
Quant à la réalité comme structure projetée: il semble que ce soit en effet une projection émanant d'un artefact, une machine didactique comparable à un ordinateur qui nous guide, nous programme et, plus généralement, nous gouverne tandis que nous agissons sans avoir conscience d'elle, à l'intérieur du monde projeté. Cet artefact, que j'appelle Zebra, a «créé» (ou seulement projeté) la réalité sous l'aspect d'un reflet fidèle de son créateur à lui, pour que celui-ci dispose d'un point de vue objectif lui permettant de se comprendre lui-même. En d'autres termes, le créateur (que Jakob Böhme, en 1616, dénomme l'Urgrund) est incité à se chercher un instrument de conscience de soi, de connaissance de soi, une opinion, une conception, une compréhension objectives de lui-même (il s'agit d'un vaste organisme vivant qui, sans ce reflet fidèle, est dépourvu d'attributs, d'aspects, raison pour laquelle il a besoin du monde empirique comme miroir dans lequel se «voir»).

Cet organisme a construit un artefact projecteur de réalité (ou démiurge, cf. Platon et les gnostiques), qui dès lors, sur son ordre, a projeté la première phase du monde tel que nous le connaissons. Cet artefact n'a pas conscience d'être un artefact; il ignore l'existence de l'Urgrund (dans le lexique compréhensible par lui, l'Urgrund «n'est pas», plutôt qu'«est»), et se prend pour Dieu, le seul vrai Dieu.

En observant notre réalité en perpétuelle évolution, I'Urgrund acquiert une compréhension de plus en plus pertinente de lui-même. II doit permettre à l'artefact projecteur de réalité de continuer à projeter une réalité en perpétuelle évolution, même si cette dernière est défectueuse et difforme (une phase après l'autre), jusqu'à ce qu'elle soit un analogue exact, véritablement exact, de l'Urgrund luimême; à ce stade, la disparité entre l'Urgrund et la réalité projetée s'abolira - alors se produira un événement stupéfiant: I'artefact, ou démiurge, sera détruit et l'Urgrund assimilera la réalité projetée avant de la transmuter en une chose ontologiquement réelle, et de rendre immortels les

êtres vivants qu'elle renferme. Ce phénomène, cette entrée de l'Urgrund dans la structure projetée par ailleurs factice, peut survenir à tout instant.

Zebra, l'artefact énergétique projecteur, est à portée de main, mais il nous a occlus, il nous maintient à distance non seulement de ses actes, mais [même] de sa présence. Il a sur nous un pouvoir immense, pour ainsi dire déterminant.

Concernant notre monde (son destin), le pronostic est excellent: c'est l'immortalité et l'injection finale de réalité une fois qu'il aura atteint le point de congruence entre l'analogue et l'Urgrund. Mais le destin de l'artefact est la destruction (à son insu). Toutefois, il n'est pas vivant au sens où nous le sommes et où l'est l'Urgrund. Nous allons vers l'isomorphisme. À l'instant précis où celui-ci sera atteint, nous nous connecterons à l'Urgrund, nous serons assimilés et pénétrés par lui, dans une étourdissante explosion de lumière: c'est le «Blitz » de J. Böhme. [Ce qui m'est arrivé en] mars 1974 ne correspond pas à cet instant-là; c'était Zebra l'artefact qui ajustait sa réalité projetée, car elle avait dévié de sa course au fil de son évolution vers l'isomorphisme avec l'Urgrund (un but inconnu de l'artefact).

Le but de la réalité évolutive projetée étant d'atteindre un état au sein duquel nous, êtres humains, serons isomorphes avec le créateur véritable, l'Urgrund qui a façonné l'artefact projecteur, une situation concrète de la plus haute importance se profile en matière de fréquence et de profondeur: bien que nous ne soyons pas encore exactement isomorphes avec l'Urgrund, on peut dire que nous portons d'ores et déjà en nous des fragments ou fractions imparfaits (mais on ne peut plus réels) de I'Urgrund. D'où la formule issue du mysticisme chrétien: «Ce qui est au-delà est au dedans. " Cela décrit la troisième et ultime phase de l'Histoire durant laquelle les hommes seront censés être gouvernés de l'intérieur. D'où cette autre formule issue du mysticisme chrétien: «Ton corps est le temple du Christ, et en toi il est ton âme. »

Dans la philosophie hindoue, l'Ātman, à l'intérieur de l'individu, est identifié à Brahman, essence et noyau de l'univers.

Ce Christ, ou Ātman, n'est pas une microforme de Zebra, l'artefact projecteur de réalité comparable à un ordinateur, mais de l'Urgrund; ainsi est-il présenté dans la religion hindoue (sous les traits de Brahman) comme situé au-delà de Maya, le voile de l'illusion trompeuse (c'est-à-dire l'apparence du monde projeté).

Les êtres humains sont déjà si près de l'isomorphisme avec l'Urgrund que ce dernier peut à présent naître au sein d'un humain. C'est l'expérience la plus primale, la plus importante qu'un être puisse connaître. La source de tout être contourne l'artefact et son monde projeté et prend vie dans l'esprit d'un être humain de ce côté-ci, et d'un autre humain de l'autre côté.

On peut en déduire à raison que l'Urgrund est d'ores et déjà occupé à pénétrer le monde de l'artefact, donc que l'instant du Blitz, selon le terme de Böhme, n'est plus très loin. Quand la microforme de l'Urgrund naîtra dans un être humain, celui-ci jouira d'une compréhension qui s'étendra au-delà des limites temporelles et spatiales du monde. Il connaîtra alors d'autres ères, d'autres identités (ou existences), d'autres lieux. La déité centrale en lui sera, litté- 
ralement, plus grande que le monde.

Pénétrant au cœur du monde projeté, l'Urgrund peut, puisque émanant d'esprits humains, assimiler ce monde et, simultanément, anéantir l'artefact projecteur au moment où est atteint l'état adéquat dans le processus évolutif (y compris celui de l'homme). L'Urgrund seul sait quand cela se produira.

L'Urgrund abolira le pouvoir que détient sur nous le monde illusoire en abolissant le pouvoir déterministe de coercition que l'artefact exerce sur nous - en éradiquant l'artefact; par son propre non-être, il annulera l'être de l'artefact. Restera une structure totalement moniste, entièrement vivante et pensante. II n'existera ni lieu, ni temps, ni état en dehors de l'Urgrund.

Le monde projeté de l'artefact n'est pas malveillant, pas plus que l'artefact lui-même. Toutefois, l'artefact est impitoyablement déterministe et mécanique. On ne peut en appeler à lui. Il exécute une tâche dont il ne perçoit pas la finalité. Aux termes de ce modèle, la souffrance a deux origines:

1. La composition mécaniste inconsidérée de la réalité projetée et de l'artefact, où règne la causalité aveugle;

2. Ce que le NT [Nouveau Testament] appelle «les douleurs de l'enfantement de l'univers " [sic], tant dans le macrocosme que dans le microcosme humain.

L'enfantement attendu est avant tout la naissance de l'Urgrund en chaque être puis, pour finir, l'assimilation de l'univers dans sa totalité en un unique instant parfaitement défini. Le premier de ces deux événements est en train de se produire; le second surviendra à l'improviste.

La réalité doit être vue comme processus. Toutefois, si la souffrance aiguë des êtres vivants qui doivent subir ce processus est indéniable, sans qu'ils en comprennent la raison, l'Urgrund se permet occasionnellement une miséricordieuse intervention qui l'emporte sur la chaîne causale de l'artefact, prévaut sur elle et l'annule. II se peut que cette intervention salvifique résulte de la naissance de I'Urgrund en l'individu. Notons que, historiquement, le mot «salut » signifie «libération », et qu'au départ, I'homme qui a «péché », qui est «déchu», est un homme «asservi ». Dans ce modèle, on peut a priori imaginer une libération de l'être humain par rapport à la domination exercée par l'artefact, aussi positive, utile et raisonnée soit son activité. Celui-ci est de toute évidence capable de commettre une erreur; il n'est pas à l'abri de l'imperfection. De toute évidence, cette prise de contrôle est parfois essentielle, tou- jours dans le cadre de ce modèle. De même, il est évident qu'en toute logique, c'est le créateur premier, principe et soubassement de l'être, qui possède la sagesse et le pouvoir nécessaires. En aucun cas une chose sise à l'intérieur de l'artefact ou émanant de lui ne pourrait y suffire.

\section{AVANTAGeS DE CE MOdÈle}

Fondamentalement, ce modèle laisse entendre que le monde empirique est, de la part d'une entité limitée, une tentative d'imitation d'un sujet qu'elle ne peut voir. Ce qui expliquerait les imperfections et éléments «malveillants» du monde.

De plus, cela explique la raison d'être du monde empirique. II est processus dirigé vers un but bien précis, qui a été défini.

Dans ce système, I'homme n'est pas accusé d'avoir provoqué la chute de la Création (il n'est pas satisfaisant d'affirmer que l'homme a provoqué la chute de la Création dans la mesure où il semble être la principale victime, et non l'auteur, des maux de ce monde). Ce système ne tient pas non plus Dieu pour responsable du mal, de la souffrance et du malheur (idée également inacceptable); au lieu de cela, il existe une troisième hypothèse: une entité limitée, dénommée «l'artefact », qui fait au mieux de ses possibilités vu ses limitations. Ainsi, il n'est pas supposé de divinité mauvaise (dualisme iranien, gnosticisme).

Bien que très complexe, ce modèle respecte le principe de parcimonie puisque, si l'on fait l'économie du concept d'artefact intermédiaire, c'est soit Dieu, soit I'homme qui est responsable de l'abondance de mal et de souffrance dans le monde, une théorie qui soulève bien des objections.

Mais par-dessus tout, ce modèle semble concorder avec les faits, lesquels semblent être:

1. Le monde empirique n'est pas vraiment réel, il ne l'est qu'en apparence.

2. Son créateur ne peut être sollicité pour rectification ou redressement de ces maux et imperfections.

3. Le monde avance vers quelque espèce d'état ou dessein final dont la nature demeure obscure, mais le caractère évolutif des étapes du changement laisse croire à un état final positif et raisonné qui a été conçu par une proto-entité pensante et bienveillante. 
Autre chose. Il y a apparemment une boucle rétroactive entre l'Urgrund et l'artefact par laquelle le premier peut exercer une pression sur le second dans certaines circonstances exceptionnelles, à savoir quand l'artefact s'écarte des séquences correctes qui meuvent le monde projeté vers l'état analogue à l'Urgrund. Soit ce dernier modifie directement l'activité de l'artefact en exerçant sur lui une pression directe, soit il rejoint le monde projeté et le module en contournant l'artefact, soit les deux. Quoi qu'il en soit, l'artefact est occlus quant à la nature et à l'existence même de l'Urgrund, comme nous-mêmes le sommes par rapport à l'artefact. Une boucle d'inconnaissance se referme, dans laquelle la source première (Urgrund) et la réalité finale (le monde) avancent vers la fusion, et l'entité intermédiaire (l'artefact) vers l'élimination. Ainsi le schéma global va vers la perfection et la simplification, en s'éloignant de la complexité et de l'imperfection.

Même si l'ajout de ce point doit avoir pour effet de complexifier le modèle, je propose la modification suivante:

Il est possible que l'Urgrund interagisse perpétuellement avec la fonction "projectrice de monde " de son propre artefact, de telle manière que le monde empirique produit soit le résultat d'une dialectique constante. Auquel cas I'Urgrund a bipolarisé l'artefact par rapport à luimême, le monde empirique étant alors à considérer comme le produit de deux forces entremêlées de type yin/ yang: l'une vivante, pensante et consciente de la situation globale, l'autre mécanique et active, mais incomplètement consciente.

Le monde empirique est dans ce cas le fruit d'un «étant » (l'artefact) et d'un «non-étant » supérieur (l'Urgrund).

Pour les êtres qui vivent dans le monde empirique projeté, il serait pratiquement impossible de distinguer les pressions émanant de l'artefact (incorrectement considéré comme maléfique) de celles émanant de l'Urgrund (correctement considéré comme bénéfique). Ils ne percevraient qu'un grand flux, un changement évolutif constant qui ne revêtirait de gestalt particulière en aucun point précis du temps linéaire.

Toutefois, ceci semble bien correspondre à ce que nous percevons du monde. Le fondement premier de l'être construit quelque chose (l'artefact) contre quoi jeter son soi propre, et il en émane le monde tel que nous le connaissons.

Cette modification du modèle expliquerait que l'artefact imite une chose qu'il ne peut voir, et dont il n'a, en fait, même pas conscience.

L'artefact considérerait sans doute les intrusions de l'Urgrund dans sa propre projection du monde comme une étrange invasion à combattre. Si bien que, plus qu'à tout autre, parmi tous les systèmes philosophiques et théologiques connus, le conflit qui en résulterait ressemblerait à celui formulé par Empédocle, avec ses oscillations du chaos opposées à la formation de krasis (gestalt) successives. En dehors d'une révélation directe de la part de I'Urgrund, nous ne pourrions qu'inférer obscurément la présence et la nature des deux forces en interaction, ainsi que l'état final proposé du monde.

Certains éléments tendent à démontrer que l'Urgrund communique effectivement ce type de révélation aux êtres humains afin de mouvoir le processus dialectique vers le but souhaité. D'un autre côté, l'artefact contrerait cette démarche en induisant le plus possible de cécité ou d'occlusion; dans cette vision, les ténèbres et la lumière semblent en guerre - ou plus exactement la connaissance et l'inconnaissance, les êtres humains se rangeant à juste titre au côté de l'entité de la connaissance (appelée Sainte Sagesse)

Toutefois, en conclusion, je suis pessimiste quant à la fréquence d'intervention de l'Urgrund ici-bas, dans le monde projeté de l'artefact. Le dessein de celui-ci (ou, plus justement, le dessein de l'Urgrund) est plutôt atteint sans intervention; ce qui revient à dire que l'isomorphisme est progressivement atteint, à un rythme régulier, en tant qu'ultime but souhaité, sans qu'aucune intervention soit nécessaire. L'artefact a été fabriqué pour effectuer une unique tâche, et il s'en acquitte avec succès.

II semble qu'une sorte d'interaction dialectique intervienne dans l'évolution de la projection, mais sans nécessairement faire intervenir l'Urgrund; il peut aussi s'agir de la méthode de fonctionnement de l'artefact seul.

Ce que nous devons espérer, attendre avec impatience, c'est le moment de l'isomorphisme avec le fondement de l'être, la réalité première qui, sous forme d'Étincelle divine, pourra naître en nous. L'intervention dans ce monde en tant que tel ne se produira qu'à la fin des temps, quand l'artefact et le règne tyrannique dans lequel il nous fait vivre, son joug de fer, sera aboli. L'Urgrund est réel, mais lointain. L'artefact est réel aussi, et proche, mais n'a ni oreilles pour entendre, ni yeux pour voir, ni âme pour écouter.

Le malheur n'a d'autre raison d'être que la voie qui permet d'en sortir et d'atteindre à la joie triomphante. Cette voie passe par la mort de l'ego des hommes, qui est alors remplacé par la volonté de l'Urgrund. Tant que ce stade ultime n'est pas atteint, chacun d'entre nous est réifié par l'artefact. Nous n'avons pas la possibilité de désavouer arbitrairement son monde, tout projeté qu'il soit, puisque c'est le seul dont nous disposons. Mais à l'instant où l'ego 
individuel meurt et où l'Urgrund naît en nous - à cet instantlà nous nous trouvons libérés de ce monde et redevenons une partie de notre source originelle. L'initiative vient de I'Urgrund; si malheureux que soit ce monde projeté, aussi insensible à la souffrance que soit l'artefact, il s'agit tout de même de la structure créée par l'Urgrund et via laquelle nous atteignons à l'isomorphisme. S'il y avait eu un meilleur moyen, l'Urgrund y aurait certainement eu recours. Le chemin est ardu, mais le but en vaut la peine.

En vérité, en vérité, je vous le dis, Vous pleurerez et vous vous lamenterez, Et le monde se réjouira;

Vous serez dans la tristesse, Mais votre tristesse se changera en joie. La femme, lorsqu'elle enfante, éprouve de la tristesse,

Parce que son heure est venue; Mais lorsqu'elle a donné le jour à l'enfant, elle ne se souvient plus de la souffrance À cause de la joie qu'elle a de ce qu'un homme est né dans le monde.

Vous donc aussi, vous êtes maintenant dans la tristesse;

Mais je vous reverrai, et votre cœur se réjouira, Et nul ne vous ravira votre joie.

(Évangile selon saint Jean, XVI, 20-22¹.)

\section{RAMIFICATIONS DE LA RÉALITÉ PROJETÉE EN MATIÈRE DE DÉNI DE PERCEPTION}

Un monde qui n'est que simple projection, et manque donc de substance ontologique, n'a pas la faculté pleine et entière de se maintenir en cas de rupture du consensus, et c'est là un défaut majeur de ce système factice. Sans le savoir, les êtres humains ont la possibilité de désavouer l'existence de cette réalité factice en lui retirant leur assentiment; mais ils sont alors contraints d'affronter les conséquences de ce qui reste, en admettant qu'il reste quelque chose.

L'idée qu'un substratum de réalité authentique non projeté, non perçu en temps normal, puisse exister sous la projection est une possibilité. II n'y a aucun moyen de mettre cette hypothèse à l'épreuve, hormis par un acte existentiel délibéré de rupture du consensus face à l'inauthentique. Une telle démarche ne peut être réalisée facilement. Elle nécessite à la fois un acte de désobéissance vis-à-vis de la projection factice et un acte de foi vis-à-vis du substratum authentique - sans qu'il y ait jamais eu perception du substratum, peut-être, sous quelque aspect que ce soit. Je postule donc qu'il faudrait qu'une entité extérieure déclenche ce double processus complexe de retrait de l'assentiment et d'adhésion simultanée à ce qui existe de manière invisible.

S'il existait un tel substratum de réalité authentique substitutif, invisible, sous l'inauthentique réalité projetée ou d'une quelconque manière masqué par elle, il constituerait la substance du plus grand savoir ésotérique imaginable. Je propose la proposition [sic] qu'il existe bel et bien un tel substratum invisible; qui plus est, je propose la proposition [sic] qu'un groupe ou une organisation secrets manie cette connaissance et ces procédés protégés de manière à déclencher une perception, si limitée soit-elle, du substratum authentique. Je vois dans ce groupe ou cette organisation l'Église chrétienne véritable, clandestine, persécutée, qui œuvre au fil des siècles de manière souterraine, en lien direct avec une gnose, des traditions et des procédés ésotériques transmis oralement depuis le Christ. Je postule par ailleurs que ce déclenchement de la prise de conscience du substratum authentique induit par l'Église chrétienne véritable et clandestine amène en définitive l'individu à trouver, pénétrer ou voir ce qui est décrit dans le NT sous le nom de Royaume de Dieu.

On peut donc dire que pour ces individus, et ceux chez qui se déclenche la prise de conscience, le Royaume de Dieu est bel et bien advenu comme il est dit dans le NT, c'est-à-dire du vivant de certaines des personnes qui ont côtoyé le Christ.

Pour finir, je propose une étonnante idée: Christ est revenu, ressuscité, peu après la crucifixion, sous l'identité de ce qu'on appelle le Paraclète, et il est capable d'induire une théolepsie qui équivaut fonctionnellement à la naissance de l'Urgrund dans la personne concernée. Enfin, j'affirme que le Christ est une microforme - et non un produit - de l'Urgrund: I'Urgrund lui-même. II n'entend pas la vox dei [voix de Dieu], il est la vox dei. II était la pénétration initiale, par l'Urgrund, de ce pseudo-monde projeté, et il n'est jamais parti.

Le substratum authentique révélé par la désobéissance et le déni du monde factice est la réalité du Christ Lui-même, l'espace-temps du Premier Avènement, autrement dit: la partie de la structure factice d'ores et déjà transmuée par la pénétration de l'Urgrund. Le Premier Avènement ayant représenté le stade initial de cette pénétration, il n'est guère étonnant qu'il en constitue encore le segment de réalité pure et authentique, bipolarisé contre la contrefaçon projetée. Sis en dehors du temps linéaire, 
en dehors de toutes les limitations du monde projeté de l'artefact, il est éternel et parfait et, en théorie, littéralement, en permanence à portée de main. Mais la rupture du consensus, de l'assentiment au monde projeté, est la condition requise pour percevoir cette réalité suprême, pour en faire l'expérience, et ce phénomène doit être induit de l'extérieur. C'est un acte de foi absolue que de désavouer le monde empirique pour affirmer la réalité vivante du Christ, c'est-à-dire Christ en nous, masqué par le pseudo-monde. Cette divulgation est le but du christianisme authentique, et est accomplie par nul autre que le Sauveur Lui-même.

La séquence est donc la suivante: la structure factice projetée qui se voit désavouée, découverte, révèle un unique modèle intemporel: Rome circa l'an 70 apr. J.-C. et les chrétiens en butte à l'État, presque une forme archétypique platonicienne, dont on retrouve les échos à travers les ères linéaires successives.

Le thème de l'assujettissement suivi du salut, ou celui de l'individu déchu puis libéré, sont issus de la figure matricielle originale du chrétien révolutionnaire luttant contre les puissantes légions de l'Empire romain. En un sens, il ne s'est plus rien produit après l'an 70 apr. J.-C. La crise archétypique est continuellement rejouée. Chaque fois qu'on défend la liberté, on retrouve les chrétiens combattant Rome; chaque fois que des êtres humains sont réduits en esclavage, on retrouve la tyrannie romaine qui s'exerce contre les êtres faibles et sans défense. Toutefois, le monde inauthentique projeté de l'artefact masque cette lutte intemporelle. La révélation de la lutte est un autre secret, que seul peut révéler le Christ en tant qu'il est l'Urgrund.

Telle est la dialectique primordiale: la libération (le salut) contre la servitude (le péché, la chute). Dans la mesure où ils asservissent les hommes sans même que ceux-ci s'en aperçoivent, l'artefact et son monde projeté peuvent être considérés comme «hostiles", à savoir voués à l'asservissement, à la tromperie et à la mort spirituelle. Le fait que même ce phénomène soit mis à profit par I'Urgrund, qui met toute chose à profit, est un secret sacré et ardu. On dira que la pénétration libératrice, par l'Urgrund, du monde projeté, est la victoire ultime et absolue de la liberté, du salut, du Christ Lui-même; la splendide résolution d'un conflit intemporel.

II existe un parallèle entre le chemin du salut et le chemin de la chute tel qu'on se le représente communément, et tel que Milton l'a ainsi évoqué:
La première désobéissance de l'homme et le fruit De cet arbre défendu dont le mortel goût Apporta la mort dans le monde, et tous nos malheurs

[...]

(Le Paradis perdu, I, $1^{2}$.)

La désobéissance est la clef du salut comme elle est la clef, on l'a vu, de la Chute primordiale (en admettant que celle-ci ait bel et bien eu lieu); mais en tant que telle, ne s'agit-il pas d'une désobéissance vis-à-vis de l'organisation actuelle des choses, laquelle organisation, si elle est bipolarisée contre l'Urgrund, est en même temps une désobéissance envers Dieu? Le talon d'Achille du monde projeté, asservissant et trompeur, est infime et difficilement accessible, mais dans le cadre de ce modèle, il peut être défini : le retour à ce qui se conçoit comme l'état divin originel de l'être humain fait pour ainsi dire son apparition par la voie de la désobéissance envers une chose qui, quel que soit le degré de coercition de son pouvoir sur nous, est une falsification. La désobéissance envers le monde projeté de l'artefact renverse, détrône au sens le plus littéral qui soit ce monde projeté à condition que la désobéissance emporte un déni de la réalité de ce monde et aussi (ceci est absolument indispensable) une postulation du Christ, plus précisément le Christ éternel et cosmique dont le corps est par essence un «monde» authentique sous-jacent à ce que nous voyons.

L'artefact, si on lui désobéit, postulera qu'il est Dieu, le Dieu légitime, et que la désobéissance est une faute contre le Créateur de l'homme et du monde. II est en effet le créateur du monde, mais non de l'Homme. L'Urgrund et l'Homme, étant isomorphes, s'opposent ensemble au monde. Tel est l'état qu'il convient d'atteindre. L'alliance est une alliance contre I'Urgrund. Dieu et l'Homme sont fait pour s'élever ensemble contre le monde projeté.

Pour postuler Dieu effectivement, il faut poser le désaveu du monde. Celui-ci, qui est doté d'une puissance matérielle colossale, peut faire planer sur les hommes qui lui désobéissent et le nient la menace du châtiment et mettre cette menace à exécution. Toutefois, nous avons reçu la promesse que nous bénéficierions d'un Intercesseur, et ce de la part du Christ Lui-même, qui sera (a déjà été) envoyé par le Père (l'Urgrund) pour nous défendre et nous réconforter - en fait, pour prendre littéralement la parole en notre faveur devant les tribunaux des hommes. 
Sans cet Intercesseur, le Paraclète, nous serions détruits dès que nous désavouerions le monde. La seule manière de démontrer la réalité de l'Intercesseur est de faire acte de foi et d'affronter le monde. II faut donc un courage énorme, car l'Intercesseur n'apparaît que si le désaveu est prononcé.

Revenons à présent à la description que j'ai donnée au départ de cet artefact comme machine didactique. De quoi nous instruit-elle? Il y a là une énigme, une espèce de jeu de casse-tête; nous sommes censés assimiler étape par étape soit une série de leçons de plus en plus difficiles, soit une unique leçon bien précise. Nous sommes confrontés au cours de notre existence à cette ou ces énigmes sous diverses formes; si nous parvenons à les résoudre, nous passons à l'étape suivante; sinon, nous restons là où nous sommes.

La toute dernière leçon apprise advient lorsque la machine didactique (ou l'Instructeur) est désavouée, reniée. Jusqu'à ce qu'arrive ce moment (s'il arrive, dans le cas de certains d'entre nous), nous demeurons sous le joug de la machine - sans même en avoir conscience, puisque nous n'avons jamais connu autre chose.

Par conséquent, cette série de leçons dispensées par l'artefact a pour but d'induire une révolte contre la tyrannie de l'artefact lui-même, et on a là un paradoxe. II sert l'Urgrund en nous amenant, à terme, à l'Urgrund. C'est ce qu'on appelle en théologie le «pacte secret», qu'on retrouve dans les religions d'Égypte et d'Inde. Des dieux qui, en apparence, se combattent mutuellement, pactisent au plan transmatériel afin d'atteindre un même but. Pour ma part, je crois que c'est le cas ici. L'artefact nous asservit, mais parallèlement, s'efforce de nous apprendre à nous libérer de ce joug. Jamais il ne nous dira de lui désobéir. Vous ne pouvez pas ordonner à quelqu'un de vous désobéir; c'est à la fois sémantiquement et fonctionnellement impossible.

1. Nous devons reconnaître l'existence de l'artefact.

2. Nous devons reconnaître le caractère factice du monde empirique engendré par l'artefact.

3. Nous devons appréhender que l'artefact, parce qu'il a le pouvoir de projeter le monde, nous a asservis.

4. Nous devons reconnaître que l'artefact, bien qu'il nous asservisse dans un monde falsifié, nous dispense un enseignement.
5. Nous devons enfin désobéir à notre instructeur - et c'est peut-être le moment le plus difficile de notre vie car il dit:

«Je vous réduirai à néant si vous me désobéissez, et je serais moralement en droit de le faire puisque je suis votre créateur. »

Fondamentalement, non seulement nous désobéissons alors à notre instructeur, mais nous nions sa réalité (en relation avec une réalité supérieure qui ne se révèle qu'au moment où se produit cette dénégation).

II s'agit là d'un jeu complexe aux enjeux suprêmes: la liberté et le retour à la source de notre être. Et chacun d'entre nous doit s'y livrer seul.

C'est un point très curieux, et que je discerne pour la première fois. Les êtres que l'artefact comble de plaisirs et de récompenses via son monde projeté ont moins tendance à prendre position contre lui et son monde. Ils ne sont pas hautement incités à lui désobéir. Mais ceux qui sont punis par lui, ceux à qui il inflige malheur et souffrance, ceux-là seront incités, pour finir, à interroger la nature de l'entité qui gouverne leur vie.

J'ai toujours eu la sensation que la raison d'être fondamentale et constructive de la souffrance était l'éveil. Mais à quoi s'agit-il de nous éveiller? Le présent essai l'indique peut-être. Si l'artefact, via son monde projeté, nous apprend à nous rebeller et si, ce faisant, nous atteignons l'isomorphisme avec notre véritable créateur, alors c'est la voie ardue qui mène à l'immortalité et au retour à la source divine. La voie des plaisirs (réussite, récompense de la part du monde projeté et à l'intérieur de lui), elle, ne nous poussera pas vers la prise de conscience, vers la vie.

Nous sommes sous le joug d'un mécanisme sans pitié qui refuse d'écouter nos doléances; par conséquent, nous le désavouons, lui et son monde, et nous nous en détournons.

La machine didactique analogue à un ordinateur accomplit bien sa tâche. C'est pour elle une tâche ingrate - et pour nous une expérience malheureuse. Mais l'enfantement n'est jamais chose aisée.

II ne peut y avoir naissance divine au sein de l'esprit humain tant que cet humain n'a pas désavoué le monde. Une fois déjà il s'est rebellé, et cela a entraîné sa chute; il lui faut donc à présent se rebeller à nouveau pour retrouver sa condition perdue. 
Ce qui a jadis causé sa perte sera son salut. II n'y a pas d'autre voie.

Le créateur est invité à rechercher un instrument de prise de conscience de lui-même: telle est la prémisse de cet essai. Et notre réalité a été construite de manière à agir comme une sorte de miroir ou de reflet de son créateur, de telle façon que celui-ci y puise un point de vue objectif susceptible de l'aider à se comprendre lui-même.

Depuis la rédaction de ceci, je suis tombé sur l'article de l'Encyclopédie de la philosophie consacré à Giordano Bruno (1548-1600). On y apprend notamment qu'il a transformé les idées d'Épicure et de Lucrèce en désignant les mondes innombrables comme des choses animées, et en déclarant que l'infini a pour fonction d'être l'image de la divinité infinie. On peut également y lire p. 407, au chapitre «Art de la mémoire», que, dans son art occulte de la mémoire, il place au-dessus de tout l'exercice intensif de l'imagination. Il perpétue ainsi une tradition typique de la Renaissance qui a également son origine dans un renouveau de l'hermétisme, car l'expérience religieuse du gnostique hermétique consiste à recréer le macrocosme dans le microcosme. Si, en effet, l'hermétique se croit capable de cet exploit, c'est que pour lui, la mens [esprit] humaine est divine per se, donc à même de reproduire en elle l'esprit divin sous le cosmos. Chez G. Bruno, le développement de cette mémoire magique reflétant le monde devient une méthode permettant de se doter d'une personnalité de mage, un mage qui se pense comme le chef d'un mouvement religieux.

Le type de mémoire que cultivait ainsi G. Bruno - et les méthodes d'enseignement destinées à rétablir cette mémoire - constituent la mémoire phylogénétique à long terme, qui s'étend sur de multiples existences humaines. Le processus de récupération de cette mémoire à long terme est l'anamnèse, mot qui signifie littéralement «perte de l'oubli ». C'est donc exclusivement via l'anamnèse que naît la mémoire véritablement susceptible de «refléter l'esprit divin sous le cosmos». Par conséquent, s'il doit accomplir sa tâche - qui est de constituer une sorte de miroir ou d'image de l'Urgrund -, l'être humain doit en passer par l'anamnèse.

Celle-ci est atteinte lorsque certains circuits neuronaux sont désinhibés. L'individu ne peut y parvenir seul; le stimulus désinhibiteur se trouve à l'extérieur de lui, et doit lui être exposé, après quoi se déclenche dans son cerveau le processus au terme duquel il deviendra un jour capable d'accomplir sa tâche.
C'est l'Église chrétienne véritable et secrète qui entre çà et là en contact avec les hommes afin de déclencher cette anamnèse - laquelle agit simultanément pour que l'homme voie le monde projeté tel qu'il est vraiment. II se trouve donc libéré par le fait même d'accomplir sa tâche divine.

Les deux règnes - 1) le macrocosme, ou univers, et 2) le microcosme, l'homme - possèdent donc des structures analogues.

1. En surface, l'univers consiste en une réalité projetée, factice, sous laquelle se trouve un substratum authentique de nature divine. II est difficile de pénétrer jusqu'à ce substratum.

2. En surface, l'esprit humain consiste en un ego à court terme, limité, qui naît, meurt et n'appréhende que très peu de choses; mais sous cet ego humain se trouve l'infinitude divine de l'esprit absolu. II est difficile de pénétrer jusqu'à ce substratum.

Mais s'il y a pénétration jusqu'au substratum divin dans le microcosme, le substratum divin du macrocosme se manifestera à l'individu.

Àl'inverse, s'iln'y a pas pénétration interne jusqu'au substratum divin dans l'individu, la réalité extérieure de ce dernier demeurera occluse par le monde factice projeté par l'artefact.

Le point d'entrée par où opérer cette transformation se trouve dans l'individu, le microcosme, et non dans le macrocosme. Là où advient la métamorphose sanctificatrice. On ne peut demander à l'univers d'ôter son masque si, de son côté, l'individu n'enlève pas le sien. Toutes les religions à mystère, y compris hermétiques, alchimiques et chrétiennes, posent l'être humain comme cible au moyen de laquelle transmuer l'univers. En changeant la personne, c'est le monde qu'on change.

Derrière l'esprit humain il y a Dieu.

Derrière l'univers falsifié il y a Dieu.

Dieu est séparé de Dieu par le factice. Abolir les strates internes et externes factices, c'est rendre Dieu à Lui-même - ou plutôt, comme l'expose cet essai, Dieu fait alors face à Lui-même, se voit Lui-même en toute objectivité, s'appréhende et se comprend enfin.

L'univers processus est un mécanisme par lequel Dieu se retrouve enfin face à Lui-même. Ce n'est pas I'homme qui est éloigné de Dieu, mais Dieu qui est éloigné de Dieu. Il a très certainement voulu dès le début qu'il en soit ainsi, et n'a jamais cherché à revenir à son point de 
départ. Peut-être peut-on dire qu'il s'est infligé à Luimême ignorance, perte de mémoire et souffrance-errance et aliénation. Mais au regard de son besoin de savoir, c'était une nécessité absolue. II ne nous demande rien qu'il ne se soit déjà demandé à Lui-même. Böhme parle de «divine agonie». Nous faisons partie de cela, mais le but, la résolution le justifie. «La femme, lorsqu'elle enfante, éprouve... » Dieu est encore à naître. Le temps viendra où nous oublierons la souffrance.

II ne sait plus pourquoi il s'est infligé tout cela. II ne s'en souvient pas. Il s'est laissé asservir, berner, contraindre et, pour finir, tuer par son propre artefact. Lui qui est vivant est à la merci de ce qui est mécanique. Le serviteur est devenu le maître et le maître le serviteur. Soit le maître renonce de lui-même au souvenir de ce qui est arrivé, comment et pourquoi, soit sa mémoire a été effacée par le serviteur. Dans un cas comme dans l'autre il est victime de l'artefact.

Mais celui-ci lui apprend laborieusement, par degrés, sur des milliers d'années, à se rappeler - qui il est et ce qu'il est. Le serviteur devenu maître s'efforce de rétablir les souvenirs perdus du maître, et donc sa véritable identité.

On pourrait avancer qu'il a construit l'artefact non pas pour l'induire en erreur, mais pour rétablir sa mémoire. Toutefois, il se peut que l'artefact se soit rebellé et n'ait pas exécuté sa mission. L'artefact le maintient dans l'ignorance.

L'artefact doit être combattu - il faut lui désobéir. Alors la mémoire reviendra. C'est un élément de la Déité (Urgrund) qui a été, d'une manière ou d'une autre, capturé par l'artefact (le serviteur); celui-ci retient désormais cet élément - ou ces éléments - en otage(s). Quelle cruauté envers eux, qui sont des fragments du maître légitime! Quand viendra le changement?

Quand les éléments retrouveront la mémoire et seront rétablis. Ils doivent d'abord s'éveiller, puis faire retour.

L'Urgrund a envoyé un Champion à notre secours. L'Intercesseur. Celui-ci est ici en ce moment même. Lorsqu'il est venu pour la première fois, l'artefact l'a détecté et éjecté. Il est invisible, excepté pour ceux qu'il secourt. L'artefact ignore que l'Intercesseur est revenu; le sauvetage s'opère furtivement. L'Intercesseur est partout et nulle part.

«Car, comme l'éclair part de l'orient et se montre jusqu'en occident, ainsi sera l'avènement du Fils de l'homme. " (Matthieu, XXIV)
Il est parmi nous, mais il ne se trouve pas en un seul lieu. Comme l'a dit sainte Thérèse d'Ávila: «Christ aujourd'hui n'a d'autre corps que le vôtre ", c'est-à-dire le nôtre. Nous sommes en passe de nous transmuer en lui. Il voit par nos yeux. Le pouvoir de tromperie décroît. L'artefact a-t-il accompli sa tâche? Peut-être sans en avoir l'intention.

Si l'«image de l'esprit divin caché sous l'univers peut effectivement se refléter dans l'esprit/la mémoire divins de l'individu ", comme l'affirme l'hermétique, alors la séparation entre le monde matériel (l'ici et maintenant) et le monde éternel (l'au-delà, le paradis) ne tient plus. Supposons un esprit polyencéphalique, ou collectif, qui s'étend à travers l'espace et le temps (transspatial et transtemporel), et auquel ont pris part des sages de toutes les époques, qu'ils soient d'influence chrétienne, hermétique, alchimique, gnostique, orphique, etc. À travers leur participation au sein de ce vaste esprit, la volonté de Dieu serait effectivement réalisée, ici, sur terre, au fil de l'histoire humaine.

Bien des gens tomberont d'accord pour dire que cet esprit de type «Déité » existe pour nous après la mort; mais qui a conscience du fait que, pour certaines personnes, il peut être rallié avant, et qu'au moment où on s'y rallie, il peut devenir la psyché de ces personnes, déterminer leurs actes et penser à leur place? C'est ainsi que la mens dei [esprit de Dieu] intervient dans les affaires humaines (et peut en outre moduler les chaînes causales). Ceci révèle un formidable secret ésotérique connu des seuls «mages» au fil des âges: les deux royaumes, le ciel et la terre, ne sont pas complètement séparés. La volonté de Dieu s'exerce ici-bas, au moins pour le moment. Et de toute évidence, c'est le cas depuis longtemps puisque les Hermétiques et autres religions à mystères remontent à l'Antiquité.

À travers le Christ, Dieu est venu ici-bas s'incarner en homme, et en cet instant la séparation entre les deux royaumes a été abolie. Quant aux humains choisis pour prendre part à cet esprit collectif, il faut nécessairement qu'ils soient immortels. II y a donc un secret encore plus profondément enfoui que [ce que j'ai] découvert jusqu'ici. Monde illusoire projeté par un artefact ex-serviteur - substratum divin sous-jacent -, voyage dans le temps - et maintenant, voilà que je postule un Corpus Christi perfectionné (selon le modèle que j'en élabore) s'étendant sur la totalité du temps et de l'espace - temporellement et spatialement ubiquitaire. Cela ressemble au noûs [esprit absolu] de Xénophane, avec l'ajout de ceci: les vivants 
peuvent y participer. Et en un sens, ce noûs est réellement le gouvernant secret du monde, de telle manière que ceux qui viennent s'y intégrer en deviennent des «terminaux" - c'est-à-dire des Christs temporaires.

Cet esprit tend vers l'Urgrund sans ligne de démarcation bien claire. À ce niveau, tout ne fait qu'un: I'homme s'élève vers la divinité en réaction à l'incarnation de Dieu en l'homme ici-bas.

II semble qu'il y ait, au sein de cet esprit collectif, interpénétration entre âmes participantes. Cet esprit s'étend sur des milliers d'années, lesquelles sont toutes maintenant de la même façon que tous les lieux sont ici (ce qui explique que je me sois retrouvé à Rome en l'an 70 apr. J.-C. et aussi en Syrie, que j'aie vu Aphrodite, etc.).

À propos de cet esprit, j'ai dit: «ll est le gouvernant secret du monde. » Ceci n'est pas son monde... en surface. Les strates superficielles sont celles d'une projection factice émanant de l'artefact. Mais au-dessous, la mens dei, y compris un certain nombre de composantes humaines (tant vivantes que sises dans l'au-delà), module cette réalité de manière invisible, en s'opposant aux intentions de l'artefact. Le substratum divin, caché, authentique, est la mens dei, au-dessous du factice.

Ce que j'ai vécu en mars 1974 peut être vu comme une fructueuse tentative de la part de l'Urgrund pour atteindre son objectif, qui est de se refléter lui-même vers lui-même, en se servant de moi comme point de réflexion. J'affirme que ce faisant il a pu, d'une manière ou d'une autre, placer en moi la totalité de lui-même (et non un simple fragment comme je l'ai dit à l'origine), sous forme d'image. Ignorant la raison pour laquelle il a été créé, l'artefact y a substantiellement contribué; d'une certaine manière, en m'infligeant une souffrance excessive il m'avait, effectivement, éveillé. Autrement dit, il avait réussi à détruire la strate de la personnalité individuelle en lui assenant une série d'afflictions auxquelles mon moi, mon ego, ne pouvait survivre. C'est ainsi que la microforme de l'Urgrund a été exposée et qu'elle a perçu sa macroforme dans la totalité de l'univers - ou, comme dit l'article [de l'encyclopédie] sur [G.] Bruno, le divin derrière l'univers.

Auquel cas ce que j'ai vécu en mars 74 n'est pas tant mon expérience que celle de l'Urgrund. C'est en fin de compte une reproduction de l'Urgrund, ici plutôt que là-bas. La totalité de la Déité s'est récapitulée en moi via un processus de dévoilement de ce qui, à l'intérieur, est permanent, par ré-enroulement des strates factices ou transitoires. On peut donc dire que j'étais l'Urgrund, en fin de compte, ou du moins un reflet fidèle de lui. Le but tout entier de l'acte de me créer moi, de créer l'univers en tant que tel ainsi que les êtres vivants qu'il contient, était atteint. Considérées sous cet angle, ma vie et celle de mes ancêtres peuvent être considérées [sic] de manière téléologique, à savoir comme progressant au fil des étapes de l'évolution pour aboutir à ce moment précis. Mon expérience de 74 n'a donc pas représenté une étape de l'évolution, mais l'étape ultime, ou le but ultime, du moins si le postulat exposé du présent essai est juste.

Ce n'est pas une question de degré au niveau du reflet; soit I'Urgrund est reflété en totalité, soit il ne l'est pas du tout. [En mars 74] le reflet a été achevé, après quoi, comme je le dis ici, l'Urgrund est né de l'univers, la séquence étant représentée de la manière suivante:

L'Urgrund crée l'artefact qui projette l'univers, lequel donne lieu aux êtres vivants, qui évoluent jusqu'au stade auquel l'Urgrund «naît » ou est reflété.

Ceci reflète la suite d'étapes qu'on trouve dans I'hindouisme. En premier vient la création par Brahma. Puis Vishnou soutient l'univers. Enfin, Shiva le détruit, par où il convient de comprendre que l'univers retourne à son origine et y est réintégré. Un cycle complet - naissance, vie, retour -s'accomplit. Lorsqu'il atteint le stade de l'évolution auquel il est capable de refléter fidèlement l'Urgrund, l'univers est prêt à être réabsorbé. Ainsi, à mon sens, la déité qui règne à présent est Shiva/Dionysos/Cernunnos/ Christ, qui nous rend à notre Urgrund, ou Père: la source de notre être.

Le fait que Shiva, le dieu destructeur, soit désormais actif indique que le cycle de la création est revenu à sa source, ou plutôt que ses êtres vivants sont prêts à regagner leur source. Shiva possède un troisième œil, l'ajna qui, lorsqu'il est tourné vers l'intérieur, lui offre la compréhension pleine et entière, absolue; mais tourné vers l'extérieur, il détruit. La manifestation de Shiva (dans le système de croyance hindou) équivaut au Jour de Colère du système chrétien. Ce qu'il faut comprendre, chez ce dieu destructeur de monde, c'est qu'il est aussi le berger des âmes. Avec l'une de ses quatre mains, dans ses représentations, il rassure: il ne saurait nuire à l'homme vertueux. C'est également vrai du Christ comme Seigneur et Juge de l'Univers. Bien que le monde (la projection factice émanant de l'artefact) ait vocation à être aboli, I'homme bon n'a rien à craindre. 
II n'en reste pas moins qu'un jugement est prononcé. La répartition de l'humanité en deux groupes par le Christ est en train de s'opérer. On retrouve les mêmes divisions dans les systèmes de croyance égyptien (tel que gouverné par Osiris et Maât) et iranien (le Sage [du Livre des Rois)]. Grâce à la lucidité totale que lui confère l'ajna, Shiva le destructeur perçoit ce qu'il doit détruire au nom de la justice. Grâce à cette lucidité totale, il perçoit également ceux qu'il doit protéger. Il est donc doté d'une nature duelle: destructeur des malfaisants, protecteur des faibles, des victimes du monde, des impuissants. Le Christ aussi possède exactement ces deux natures en tant que Juge Divin et en tant que Bon Berger. Quant à Cernunnos, c'est un dieu à la fois guerrier et thaumaturge.

Pour les humains, il est difficile de concilier ces deux attributs au sein d'une seule et unique déité. Toutefois, il est possible de comprendre ce phénomène pourvu qu'on y prête suffisamment attention.

Le monde projeté de l'artefact commence à jouer son ultime et seul véritable rôle. Maintenant que l'artefact est sur le point d'être détruit, ce monde-là va finir; il n'a jamais été réel. (Ceci reflète la qualité de destructeur attribuée à Christ/Shiva/Dionysos.) Cependant, les éléments de ce monde qui ont accompli leur tâche seront retenus - c'est-à-dire sauvés - de la même manière que Dionysos est décrit comme le protecteur des petites bêtes sauvages sans défense. Dionysos est le destructeur des prisons, des tyrans, le sauveur des humbles, des faibles. Ces attributs sont justement assignés à Shiva/Cernunnos/Christ/Dionysos en raison de la nature de la tâche qu'ils ont à accomplir: une tâche jumelle, l'une consistant à détruire, l'autre à assurer le salut.

Lorsque le Fils de l'homme viendra dans sa gloire [...] il s'assiéra sur le trône de sa gloire. Toutes les nations seront assemblées devant lui. II séparera les uns d'avec les autres, comme le berger sépare les brebis d'avec les boucs; et il mettra les brebis à sa droite, et les boucs à sa gauche. Alors le roi dira à ceux qui seront à sa droite: «Venez, vous qui êtes bénis de mon Père; prenez possession du royaume qui vous a été préparé dès la fondation du monde. » [...] Ensuite il dira à ceux qui seront à sa gauche: «Retirez-vous de moi, maudits; allez dans le feu éternel qui a été préparé pour le diable et pour ses anges. "

(Évangile selon saint Matthieu, XXV-XXXI, 42.)
J'ai inféré ces caractères duels de la déité à partir de la situation elle-même. Celle-ci nécessite 1) la destruction de ce que le Christ appelle le monde «hostile» et 2) la protection des âmes méritantes. Ceci posé, on peut comprendre que la nature duelle de la déité régnante soit nécessaire. Le chapitre xxv de l'Évangile selon saint Matthieu précise clairement que cet ultime et grand jugement n'est en rien arbitraire. Qui peut remettre en cause le critère de séparation entre ceux qui trouvent place à la main gauche et à la main droite du Christ?

Alors le roi dira à ceux qui seront à sa droite [les brebis qui seront sauvées]: «Car j'ai eu faim, et vous m'avez donné à manger; j'ai eu soif, et vous m'avez donné à boire; j'étais étranger, et vous m'avez recueilli; j'étais nu, et vous m'avez vêtu; j'étais malade, et vous m'avez visité; j'étais en prison, et vous êtes venus vers moi. " Les justes lui répondront: "Seigneur, quand t'avons-nous vu avoir faim, et t'avons-nous donné à manger; ou avoir soif, et t'avons-nous donné à boire? Quand t'avons-nous vu étranger, et t'avons-nous recueilli; ou nu, et t'avons-nous vêtu? Quand t'avonsnous vu malade, ou en prison, et sommes-nous allés vers toi? » Et le roi leur répondra:

"Je vous le dis en vérité, toutes les fois que vous avez fait ces choses à l'un des plus petits de mes frères, c'est à moi que vous les avez faites. ”

Ensuite il dira à ceux qui seront à sa gauche: «[...] Car j'ai eu faim, et vous ne m'avez pas donné à manger; j'ai eu soif, et vous ne m'avez pas donné à boire; j'étais étranger, et vous ne m'avez pas recueilli; j'étais nu, et vous ne m'avez pas vêtu; j'étais malade et en prison, et vous ne m'avez pas visité. " Ils répondront aussi: «Seigneur, quand t'avons-nous vu ayant faim, ou ayant soif, ou étranger, ou nu, ou malade, ou en prison, et ne t'avons-nous pas assisté? » Et il leur répondra: "Je vous le dis en vérité, toutes les fois que vous n'avez pas fait ces choses à l'un de ces plus petits, c'est à moi que vous ne les avez pas faites. " Et ceux-ci iront au châtiment éternel, mais les justes à la vie éternelle.

(Évangile selon saint Matthieu, XxV-XxxV, 47.)

Ces formulations très imagées sont une caractéristique majeure du Premier Avènement du Christ comme déité régnante. Impossible de se méprendre sur ce chapitre de Matthieu. Les «brebis » ne s'entendent pas seulement 
dire qu'elles seront jugées: elles apprennent le critère de jugement. L'homme qui trouverait ce critère injuste se serait déjà avéré incapable de recevoir la parole divine, il serait égaré, car la décision se fonde sur le motif le plus noble et le plus sage. Toutefois, ceux qui ne verraient en Christ que le «gentil Jésus doux et humble » ferment les yeux sur son autre aspect. L'Urgrund, dont le Christ est une microforme, contient des contraires absolus. C'est pour des raisons telles que celle-ci que l'Urgrund a mis en branle un mécanisme qui lui permette de se «voir» lui-même, de se faire face, de se jauger (s'appréhender). II contient toute chose. Sans ses multiples miroirs réflecteurs, il est essentiellement inconscient (l'inconscient humain contient des contraires; la conscience est un état dans lequel ces bipolarités sont séparées, une moitié de chacune des deux étant refoulée, l'autre exprimée). C'est nous qui, en tant que miroirs, rendons de ce fait l'Urgrund conscient - ou, comme dit l'hindouisme à propos de Brahman: «Tantôt il sommeille, tantôt il danse. " Nous avons été construits pour amener l'Urgrund à l'éveil, et dès l'instant où nous accomplissons l'anamnèse, et reflétons donc fidèlement la totalité de l'Urgrund, nous amenons celui-ci à la conscience. Nous accomplissons par là, à son bénéfice, une tâche majeure - indispensable. Toutefois, une fois que nous l'avons accomplie, il nous protège et nous soutient pour toujours; il ne nous fera plus jamais défaut. Le Christ, dans les propos rapportés au chapitre xxv de Matthieu, énonce clairement que la tentative seule suffit (sans qu'il y ait considération d'un but-seulement l'amour, la bienveillance et le soutien dont est capable l'humanité). Ce qui n'est pas appréhendé (alors que le sens du passage en question est évident), c'est que les pauvres, les affamés, les malades, les étrangers, ceux qui sont nus ou emprisonnés... sont tous des formes de la déité régnante, ou doivent du moins être traités comme tels. Agir pour vêtir, nourrir, abriter, soigner et réconforter... ces acteslà constituent autant de reflets de l'Urgrund renvoyés vers lui-même. Ces actes sont l'Urgrund rendu pluriel, qui prend soin de lui-même sous ses formes diverses. II n'est pas d'acte, si infime soit-il, qui soit insignifiant. Nous connaissons le critère de jugement, nous savons quelles en sont les conséquences définitives (les métaphores du type «feu éternel, châtiment éternel » sont simplement là pour indiquer qu'une fois rendu, le jugement est sans appel; c'est du sort ultime de l'univers qu'il est question).

Quelle objection apporter à cela? Le critère de jugement est-il défaillant? En termes simples, le Christ viendra à nous sous une apparence trompeuse, verra comment nous le traitons alors que nous ne le reconnaissons pas, et nous traitera en conséquence. Le savoir devrait instiller en nous le plus haut degré d'éthique. II s'est identifié aux plus humbles d'entre nous. Qu'attendre de plus de la déité qui déterminera notre sort ultime par son jugement?

La pénétration de l'Urgrund, la déité, a atteint la strate la plus basse du monde; l'ordure dans le caniveau, le rebut, vivant ou inanimé. Depuis ce niveau inférieur, elle nous évalue, mais cherche aussi à nous venir en aide. Conformément à ses dires («La pierre qu'ont rejetée ceux qui bâtissaient est devenue la principale de l'angle »), la déité est avec nous - de la manière et dans les lieux les plus inattendus. Il y a là un paradoxe: si nous voulons Le rencontrer, il faut Le chercher là où on s'attend le moins à Le trouver. Autrement dit, là où on n'aurait jamais l'idée d'aller voir. Par conséquent - puisque cela suppose vraiment une barrière absolue -, c'est lui qui nous trouvera et non pas nous qui le trouverons.

Le Christ psychopompe, guide de l'âme, est en passe de nous ramener au bercail, de nous montrer la voie. II n'est pas là où nous le croyons; il n'est pas ce que nous croyons. Lorsqu'il prend publiquement la parole pour la première fois, à la synagogue de Nazareth, il lit ce passage d'Ésaïe:

[...] il m'a oint pour annoncer une bonne nouvelle aux pauvres, II m'a envoyé pour guérir ceux qui ont le cœur brisé,

Pour proclamer aux captifs la délivrance, Et aux aveugles le recouvrement de la vue, Pour renvoyer libres les opprimés [...]

(Livre d'Ésaïe, LXI, 1, cité in l'Évangile selon saint Luc, IV, 18-19.)

Mais comme il s'agit du Premier et non du Second Avènement, il omet un vers:

Et un jour de vengeance de notre Dieu.

(Livre d'Ésaïe, vı, 1-2.)

Le Christ du Premier Avènement ne sera plus le même lors du Second, et le vers manquant sera accompli.

II est effrayant, bien sûr, de se rendre compte que la déité vers laquelle nous nous tournons pour chercher une protection (le Christ comme berger et comme Intercesseur) est censée être le destructeur de l'univers. Mais ce qu'il faut comprendre, c'est que l'univers (ou cosmos, ou monde) a été créé dans des buts spécifiques et qu'une 
fois ces buts atteints il sera aboli, il doit être aboli, en fait, pour que soit instaurée la séquence suivante de buts à atteindre. Si on garde à l'esprit le fait que nous sommes séparés de l'Urgrund par le monde, on n'a pas de raison de se voiler la face quant à son caractère transitoire et illusoire, les deux étant liés.

Pour moi, l'Urgrund a d'ores et déjà pénétré jusque dans la strate la plus basse de ce monde illusoire projeté; théoriquement, cela fait donc de moi un panenthéiste acosmique. En ce qui me concerne, il n'y a rien de réel que l'Urgrund, tant dans sa macroforme (Brahman) que dans ses microformes (les Ātman en nous). Jakob Böhme a eu sa première révélation en surprenant le reflet du soleil sur un plat en étain. Ma révélation originelle s'est produite comme je posais les yeux par hasard sur un pendentif en or représentant un poisson, en plein soleil; je me suis entendu répondre, après m'être enquis de sa signification, qu'il s'agissait d'un «symbole utilisé par les premiers chrétiens". Ma révélation la plus récente a eu lieu devant un sandwich au jambon. Je me suis rendu compte que les deux tranches de pain de mie étaient identiques (isomorphes), mais séparées par la tranche de jambon. J'ai compris, par analogie, qu'une des deux tranches était I'Urgrund macrocosmique, que l'autre était nous, et que nous sommes une seule et même chose - avec le monde comme séparation. Enlevez le monde et les deux tranches, c'est-à-dire l'homme et l'Urgrund, deviennent une entité unique. Elles ne sont pas seulement appliquées l'une contre l'autre; elles sont une seule et même entité.

Ce monde contient beaucoup de belles choses, et il y aura de la tristesse à les voir disparaître; mais ce sont des reflets imparfaits d'une divinité qui, elle, durera éternellement. Nous sommes des étrangers dans ce monde (ici, le Christ s'adresse aux Apôtres):

\section{[...] ils ne sont pas du monde, comme moi} je ne suis pas du monde.

(Évangile selon saint Jean, XVII, 14-15.)

\section{Si le monde vous hait,}

Sachez qu'il m'a haï avant vous.

Si vous étiez du monde,

Le monde aimerait ce qui est à lui;

Mais parce que vous n'êtes pas du monde,

Et que je vous ai choisis du milieu du monde,

À cause de cela le monde vous hait.

(Évangile selon saint Jean, Xv, 18-19.)

\section{S'adressant aux Juifs, Jésus dit:}

Vous êtes d'en bas; moi, je suis d'en haut. Vous êtes de ce monde; moi, je ne suis pas de ce monde.

(Évangile selon saint Jean, VIII, 23.)

Ceux qui sont des répliques du Christ sont des répliques de l'Urgrund, et l'Urgrund est au-delà du monde, même si, depuis le Premier Avènement, invisible, il pénètre le monde. S'il était le créateur du monde il ne s'opposerait pas à lui (comme l'a exprimé le Christ); il ne serait pas contraint d'y pénétrer ainsi de manière furtive. Ces propos du Christ confirment que le monde $n$ 'est pas le produit de la Déité, mais, d'une manière ou d'une autre, son antagoniste. Les Églises bien établies de par le monde énoncent, bien sûr, une autre doctrine, puisque ce sont des artefacts, des entités de ce monde; il faut s'y attendre. On ne peut demander à une organisation issue de l'ordre régnant de nier cet ordre régnant - ainsi que les cathares l'ont constaté quand on s'est mis à les exterminer.

Si vous désobéissez au monde, le monde vous défie en vous traitant en étranger hostile, en vous percevant comme un étranger qui lui est hostile. Ainsi soit-il. Le Christ a clairement exposé la situation dans les Évangiles synoptiques.

L'ennemi de ma vie, de la justice, de la vérité et de la liberté, c'est l'irréel, l'illusion trompeuse. Le monde est une projection trompeuse émanant d'un artefact qui ne sait même pas qu'il est un artefact, ni dans quel but il projette le monde. Quand il s'en ira, ce sera soudainement, sans avertissement.
Voyez quel amour le Père nous a témoigné, Que nous soyons appelés enfants de Dieu, Et que nous le soyons en effet!
Si le monde ne nous connaît pas, c'est qu'il ne l'a pas connu.
Mes bien-aimés, nous sommes maintenant enfants de Dieu,
Et ce que nous serons un jour n'a pas encore été manifesté;
Mais nous savons qu'au temps de cette manifestation,
Nous lui serons semblables,
Parce que nous le verrons tel qu'il est.
(Première Épître de saint Jean, III, 1-2.) 
Le constructeur (de l'artefact projecteur de monde) est ici, dans les rebuts vivants de ce monde, et ses souvenirs ont été effacés, si bien qu'il n'a plus connaissance de sa propre identité. II peut être n'importe lequel d'entre nous, ou bien un certain nombre d'entre nous éparpillés çà et là. L'artefact, qui n'a pas conscience de lui, ne sait pas qu'il est un artefact, ignore sa raison d'être et finira par faire peser trop de souffrance sur ce constructeur amnésique qui se trouve ici - cet ultime excès de souffrance inutile, imméritée, infligée à l'être vivant qui, à l'insu de l'artefact et de lui-même, le constructeur, provoquera l'anamnèse soudaine; alors le constructeur «reviendra à lui-même », se rappellera qui il est et ce qu'il est - après quoi il ne fera pas que se rebeller contre l'artefact et son monde empli de souffrance: il enverra un signal à la déité régnante, Shiva, afin qu'elle détruise l'artefact et, avec lui, son monde projeté.

L'artefact ne comprend pas le risque qu'il court en infligeant une souffrance imméritée à ses créatures vivantes. Il les imagine toutes à sa merci et sans recours. En ceci, il se trompe, il se trompe absolument. Enseveli ici, mélangé à la masse indifférenciée, sans que lui-même s'en doute, existe ici l'Urgrund, avec toute la puissance et toute la sagesse que cela implique. L'artefact avance en terrain dangereux: il est de plus en plus près de réveiller son propre constructeur.

On trouve une proto-narration de ce thème dans Les Bacchantes d'Euripide. Un étranger entre dans le royaume du «Roi des larmes », qui le fait emprisonner sans motif. Cet étranger s'avère être le grand prêtre de Dionysos, quasi équivalent du dieu lui-même. II fait éclater la prison (symbole de ce monde qui asservit) puis détruit méthodiquement le roi en lui faisant perdre la raison et en rendant la chose publique de telle manière que non seulement il le détrône, mais il en fait [également] la risée de la multitude qu'il a opprimée par son règne. Si la prison en question symbolise ce monde, que représente le «Roi des larmes»? Rien de moins que le créateur de ce monde: l'artefact lui-même, mécanique, indifférent et sans scrupule, c'est-à-dire le roi ou dieu de ce monde. Le «Roi des larmes" ne soupçonne pas l'existence de la véritable nature de l'étranger qu'il a emprisonné. II ne sait pas non plus à qui l'étranger peut faire appel.

On retrouve des échos de cette proto-narration dans les Évangiles synoptiques, Ponce Pilate étant le «Roi des larmes » et le Christ l'étranger (à noter que le Christ est lui aussi originaire d'une autre province). Toutefois, contrairement à l'étranger des Bacchantes, le Christ n'a pas recours au pouvoir auquel il peut faire appel (celui du saint Père) ; cependant, lorsque le Christ réapparaîtra, cette fois il fera bel et bien appel à ce pouvoir, qui détruira l'ordre des choses tout entier, le monde comme les malfaisants. La différence essentielle entre Les Bacchantes et le Premier Avènement est que le Christ, lui, vient une première fois avertir le monde et les malfaisants, avant de réapparaître dans son rôle de destructeur. II nous laisse une chance de nous repentir, c'est-à-dire d'entendre l'avertissement.

Une comédie hollywoodienne des années cinquante montre la situation suivante: le roi d'une contrée de type médiéval, désormais trop âgé et affaibli pour gouverner, confie le pouvoir à un régent. Celui-ci, cruel et brutal, opprime la population du royaume à l'insu du vieux roi. Dans le film, un voyageur temporel venu du futur persuade ce dernier d'endosser des vêtements de paysan et, ainsi déguisé, de parcourir son royaume afin de voir comment sont traités ses sujets. Le vieux roi lui-même sera brutalement maltraité par les troupes du régent; de fait, le voyageur temporel et lui seront même emprisonnés sans raison. Après bien des péripéties, le roi réussira à s'évader et à regagner son palais, où il revêtira comme de juste ses habits royaux et révélera sa véritable identité au maléfique régent. Celui-ci sera déposé, et la tyrannie infligée aux populations innocentes sera abolie.

Conformément au modèle cosmologique proposé dans cet essai, I'Urgrund, ultime constructeur et noûs, est secrètement présent dans ce monde factice et cruel. Ignorant cet état de fait, l'artefact projetant le monde falsifié peut continuer indéfiniment à infliger en toute indifférence la vaine souffrance engendrée par la machinerie sans cervelle (autrement dit, les processus causaux) qu'il emploie et a toujours ordinairement employée. À mon sens, l'Urgrund s'est différencié à partir de l'Un qu'il était pour accéder à la pluralité. Certaines de ses parcelles ou «images » sont certainement conscientes de leur identité; d'autres sans doute pas. Mais à mesure que se maintient (voire s'élève) le degré de souffrance injustifiée, ces «images » de l'Urgrund vont se ressouvenir ensemble pour aboutir à une résurrection consciente, ce qui équivaut à une sentence de mort pour l'artefact - le «régent».

Ceci nous fournit une autre illustration de ce que saint Paul appelle les «douleurs de l'enfantement » de l'univers. La souffrance est un prélude à la naissance; dans ce cas précis, il ne s'agit pas de la naissance d'un être humain, mais de Dieu. Puisque c'est l'être humain qui subit cette souffrance, on peut en déduire que la naissance de Dieu (l'Urgrund) va se produire en l'être humain lui-même. 
L'humanité est donc, en tant qu'espèce, une Mater Dei une mère de Dieu; c'est là un concept extraordinaire aux termes duquel l'évolution biologique sur cette planète serait un moyen d'amener à l'existence un hôte ou une matrice dont Dieu Lui-même naîtrait enfin. Il est intéressant de noter qu'on trouve des arguments en faveur de cette thèse dans les Écritures: dans le NT, l'Esprit-Saint est considéré comme une divinité fécondante; I'Esprit-Saint a engendré le Christ, et le Christ redevient l'Esprit-Saint par fécondation au moment de la résurrection. L'espèce humaine revêt une nature yin, ou féminine, l'Esprit-Saint étant le yang, le principe masculin. Par conséquent, au terme de son processus évolutif l'être humain ne devient pas Dieu, mais une matrice ou un hôte destiné à accueillir Dieu; ce qui constitue une différence fondamentale. L'anamnèse est la naissance; par essence, la progéniture est née de deux parents: un être humain et l'Esprit-Saint. Sans la pénétration de l'Esprit-Saint à l'intérieur de l'être humain, l'événement ne peut se produire. L'Esprit-Saint est, bien sûr, le pons dei. Le lien entre les deux royaumes.

Chez toutes sortes d'êtres vivants existe un instinct très puissant appelé «homing " ou «instinct de retour». Citons par exemple la remontée du saumon à bosse, à partir de l'océan, vers son lieu de frai exact en rivière. Par analogie, on peut dire que l'être humain possède possiblement - et, si cela se trouve, à son insu - un même instinct du retour. Ce monde n'est pas son monde. Son vrai monde se trouve dans la région des cieux que les anciens appelaient plérôme. Le terme apparait dans le NT, mais son sens est obscur puisqu'au départ il signifie "pièce recouvrant un trou "; dans le NT il s'applique au Christ, décrit comme étant la «plénitude de Dieu», ainsi qu'aux croyants qui atteignent cette plénitude par l'intermédiaire de leur foi en Christ. Chez les gnostiques, cependant, le mot revêt une signification plus précise: il désigne la région sublunaire des cieux dont provient la connaissance secrète qui apporte à l'homme le salut.

Dans la cosmologie présentée ici, le plérôme est conçu comme étant l'Urgrund, ou la partie de l'Urgrund dont nous sommes originaires et à laquelle (si tout se passe bien) nous finirons par revenir. Si l'on considère la totalité de l'être comme un organisme qui respire (qui inhale et exhale, ce qu'on appelle harmonie palintrope), alors on peut dire - métaphoriquement - qu'à l'origine nous avons été “exhalés" par le plérôme, que nous marquons une pause transitoire sous forme de stase externalisée (notre existence ici-bas) puis que nous sommes réinhalés dans le plérôme. Ceci est la pulsation normale de la totalité de l'être, son activité fondamentale, ce qui indique qu'elle est vivante.
Un jour, sous l'effet du LSD, j'ai écrit en latin: «Je suis le souffle de mon créateur, et tandis qu'il exhale et inhale, je vis. " En tant que nous résidons ici, dans ce monde projeté, nous nous trouvons à l'état «exhalé » - nous sommes exhalés du plérôme pour une durée limitée. Toutefois, le retour n'est pas automatique; il nous faut d'abord faire l'expérience de l'anamnèse. Mais la cruauté de l'artefact est telle que selon toute probabilité, l'anamnèse sera de plus en plus hors de portée. À l'extrémité de la misère se trouve l'essence de la libération - j'ai eu cette libération, un jour, et dans cette révélation «libération » équivalait à «joie».

Que peut-on dire en faveur de la souffrance qui accable les créatures de ce monde? Rien, rien sinon qu'elle finira, de par sa nature même, par déclencher la révolte ou la désobéissance, lesquelles conduiront au retour au sein de la Déité. C'est le caractère gratuit, injustifié, de la souffrance qui en lui-même incite à la rébellion, amène à comprendre qu'il y a en ce monde quelque chose de terriblement, terriblement défaillant. Le fait même que cette souffrance soit sans objet, aléatoire et imméritée finit par la mener à sa propre destruction - et à celle de son auteur. Plus son inutilité nous apparaît clairement, plus nous inclinons à nous révolter contre elle. Si l'on tente de trouver une raison d'être, une capacité de rédemption à cette souffrance, plus on se met étroitement à la merci d'un ordre des choses impitoyable et irréel, plus on se remet entre les mains d'un tyran brutal qui n'est même pas un être vivant. Notre attitude doit être: «Je n'accepte pas ceci. Je n'y vois pas de but, de raison d'être. " Nous examinons alors cet état de fait avec une détermination infaillible, nous le répudions et, par là, nous contribuons à la répudiation de toute forme d'illusion trompeuse. Quiconque conclut un pacte avec la souffrance montre qu'il a succombé à l'artefact et en est désormais l'esclave. Avec lui l'artefact a fait une nouvelle victime, par l'obtention de son consentement. Telle est sa suprême victoire. La victime contribue à sa propre souffrance, elle accepte de contribuer à l'acceptation, au consentement au prétendu caractère «naturel » de la souffrance en général. Chercher une raison d'être à la souffrance revient à chercher celle d'une fausse pièce de monnaie. Le «but» est évident: c'est un subterfuge, une duperie. Si cette duperie nous conduit à croire que la souffrance a - doit avoir - une juste finalité, c'est que la falsification a réussi à se faire passer pour vraie et a accompli son cruel dessein. 
Dans un des Évangiles (je ne sais plus lequel), on présente un infirme au Christ et on lui demande: "Cet homme est-il infirme à cause de ses péchés, ou de ceux de son père? " À quoi le Christ répond: "Ni l'un ni l'autre. L'unique dessein ici est dans l'amélioration de sa condition, qui démontre la pitié et la puissance divines.”

La pitié et la puissance divines s'opposent à la souffrance; le NT le dit en toutes lettres. Les guérisons miraculeuses du Christ sont là pour indiquer matériellement que le Royaume de Justice est arrivé; les autres miracles ne signifient rien, ou peu de choses. Si la pitié et la puissance de l'Urgrund s'opposent à la souffrance (la maladie, la perte, la blessure) comme il est bien dit dans les Évangiles synoptiques, alors l'homme doit, s'il veut se ranger avec l'Urgrund, se dresser contre le monde, le monde dont provient la souffrance. Jamais il ne doit considérer la souffrance comme une émanation ou une initiative de la Déité; s'il commettait cette erreur de raisonnement, il se rangerait du côté du monde, donc contre Dieu. Au fil des siècles, une grande partie de la communauté chrétienne a été victime de ce piège conceptuel; sans s'en rendre compte, en encourageant ou en acceptant volontiers la souffrance, ces croyants se placent toujours plus fermement sous le joug de l'artefact.

Le fait que Jésus ait détenu le pouvoir miraculeux de guérir, mais n'en ait pas fait usage pour guérir tout un chacun, a laissé les gens perplexes, à l'époque. Luc en parle:

Je vous le dis en vérité: il y avait plusieurs veuves en Israël du temps d'Élie, lorsque le ciel fut fermé trois ans et six mois et qu'il y eut une grande famine sur toute la terre; et cependant Élie ne fut envoyé vers aucune d'elles, si ce n'est vers une femme veuve, à Sarepta, dans le pays de Sidon. II y avait aussi plusieurs lépreux en Israël du temps d'Élisée, le prophète; et cependant aucun d'eux ne fut purifié, si ce n'est Naaman le Syrien.

(Évangile selon saint Luc, Iv, 25-27.)

Piètre explication, qui expose un «quoi » et non un «pourquoi». Ce que nous exigeons c'est un «pourquoi». Mieux, nous demandons «Pourquoi pas? » Pourquoi Dieu n'abolit-il pas la souffrance s'il en a le pouvoir? On devine là-dessous une sinistre possibilité. Cela a à voir avec le pouvoir de l'artefact. Le serviteur a pris la place du maître, et il se peut qu'il possède une grande force. Une idée qui fait froid dans le dos. Shiva, dont le rôle est de le détruire, est peut-être décontenancé. Je l'ignore. Et depuis des milliers d'années, personne n'a pu apporter de réponse satisfaisante. Je propose que d'ici là, nous rejetions toutes les autres. Si nous sommes dans l'ignorance, ne le disons pas.

II me vient à l'esprit une hypothèse fondée sur une chose que j'ai vue en 1974 et que, dans leur écrasante majorité, les autres gens n'ont pas vue. J'ai pris conscience du fait que la sagesse et la puissance de l'Urgrund étaient activement à l'œuvre pour améliorer notre situation en intervenant dans le processus historique. Par extrapolation, j'en déduis que d'autres interventions, invisibles, ont dû se produire à notre insu. L'Urgrund n'avise pas l'artefact de sa présence. Supposons que l'Urgrund raisonne-à juste titre - en ces termes: si l'artefact savait qu'il est revenu pour la seconde fois, il élèverait au maximum son degré de cruauté. Nous vivons une subtile invasion qui a lieu en cachette; j'ai déjà mentionné ce fait. Une amélioration massive révélerait la présence de l'Urgrund, de la même manière que les miracles du Christ ont fait de lui une cible à l'époque du Premier Avènement. Les guérisons miraculeuses sont les lettres de crédit du sauveur et un signe de sa présence.

Si l'on pose l'existence d'un puissant adversaire à l'Urgrund, un adversaire si colossal qu'il peut projeter et maintenir en place tout un univers factice, on met aussi en évidence la preuve éventuelle de la nécessité, pour l'Urgrund, d'agir en cachette. Ses activités dans ce monde ressemblent à l'avancée à couvert d'une révolution secrète et déterminée s'opposant à une puissante tyrannie. L'Urgrund œuvre en vue d'enjeux suprêmes. II ne veut rien moins qu'annihiler ce monde et son auteur. Vraiment, je ne sais pas. J'imagine sa torture à l'idée de devoir réduire l'aide qu'il apporte aux êtres dans le besoin, mais il faut pourtant qu'il l'emporte sur l'artefact. II vise l'ennemi au cœur (ou là où se trouverait son cœur s'il en avait un), et une fois cette cible atteinte, tous les éléments, toutes les polyformes de la souffrance, de part et d'autre de la création, seront spontanément soulagés.

Peut-être en est-il ainsi ; peut-être pas. En 1974 je l'ai vu viser le centre de la tyrannie dans ce pays, les USA, et, son assaut ayant réussi, les moindres maux sont tombés en ruine un par un. L'Urgrund voit probablement ce monde factice comme une gestalt, il voit les maux polyformes provenir d'une «Quelle» - une source, en allemand. Pointer sa flèche vers la Source est une méthode de guerrier, et sous sa cape de douceur, Dieu notre sauveur est un guerrier. Tout ceci n'est que conjecture. II se peut qu'en réalité (au sens propre du terme), il n'ait qu'une seule et unique flèche à décocher. II faut qu'elle atteigne 
sa cible, faute de quoi rien n'est accompli; faute de quoi tout autre remède, toute autre amélioration seraient réduits à néant par l'artefact qui lui survivrait. L'Urgrund a clairement perçu son adversaire, ce qui n'est pas notre cas; par conséquent, il voit clairement la tâche qui lui revient et pas nous. Tout un immeuble est en flammes et nous, nous demandons aux pompiers d'arroser la fleur qui meurt. Faut-il qu'ils détournent en effet la puissance de leur jet vers la fleur? N'a-t-elle pas son importance? L'Urgrund se torture peut-être à ce propos - délaisser la fleur pour la scène dans son ensemble. Nombreux sont les humains qui ont vécu cette souffrance, et devraient donc la comprendre. N'oublions pas que I'Urgrund est ici, lui aussi, qu'il souffre avec nous. Tat twam asi [Tu es cela]. Nous sommes lui, et il doit s'extraire.

En vérité, la souffrance que nous ressentons en tant qu'êtres vivants est celle de l'éveil. Présentée ainsi, cette proposition rend compte d'un des aspects les plus désolants de la souffrance: le fait que nous soyons obligés de la subir sans savoir pourquoi. Et si nous ne savons pas pourquoi, c'est précisément parce qu'en tant que pluriformes de l'Urgrund, nous sommes encore presque inconscients. Ce serait un paradoxe si une entité inconsciente avait conscience d'elle-même et des motifs de sa situation. Distinguer la cause de notre souffrance équivaudrait à nous réveiller pleinement. Ce sera peut-être la toute dernière chose que nous apprendrons.

À ce stade, l'analogie entre artefact et machine didactique ne tient plus. Ce n'est pas une leçon que la machine (si c'est bien de cela qu'il s'agit) peut nous enseigner, puisqu'elle-même ne connaît pas l'explication. Mais en tant qu'images pluriformes de l'Urgrund, nous-mêmes nous connaîtrons a priori la raison de la situation dans laquelle nous nous trouvons quand nous accéderons au niveau requis de conscience; nous nous remémorerons. La connaissance de cette sorte repose dans nos circuitsmémoire à long terme, intrinsèques et inhibés.

Considérée comme une énigme que nous ne sommes pour l'instant pas en mesure de résoudre, la raison de notre condition souffrante (qui s'étend à tous les êtres vivants)... l'énigme en question est peut-être bien l'ultime stade de la connaissance retrouvée. S'il y a effacement de la mémoire, on peut seulement supposer qu'au moment de surmonter l'effacement ultime, nous percerons le mystère de cette inconcevable perplexité. D'ici là, la pression exercée par cette souffrance nous incite à chercher une réponse, ce qui revient à dire que cela nous pousse vers un degré de conscience de plus en plus élevé. Ce qui ne veut pas dire que la «raison d'être» de la souffrance est de produire une intensification de la conscience; cela signifie seulement qu'il en résulte une conscience qui s'intensifie peu à peu.

Quand nous serons à même d'expliquer la souffrance omniprésente chez les êtres vivants, alors nous aurons, j'en suis sûr, pleinement récupéré notre mémoire perdue; chacun aura retrouvé son identité perdue. Sommes-nous responsables de notre propre condition? Nous a-t-elle au contraire été imposée? Une des hypothèses les plus troublantes, celle de gnostiques, est que la Chute originelle (et de là la création qui, dans ce modèle, tombe sous la coupe de l'artefact projecteur de monde) n'est pas due à une faute morale, mais à l'erreur conceptuelle consistant à confondre monde phénoménal et monde réel. Cette hypothèse s'écarte de ma propre proposition, où le monde est une projection factice; le prendre pour une chose ontologiquement réelle serait en effet une affreuse erreur conceptuelle. C'est peut-être cela, l'explication. Nous nous sommes empêtrés dans un enchantement, une maisonnette en pain d'épice, comme dans les contes, qui nous a envoûtés jusqu'à nous conduire à l'asservissement et à la ruine. II se peut qu'un des postulats majeurs de ma construction cosmogonique-cosmologique soit erroné; I'Urgrund n'a pas créé l'artefact, il s'est laissé piéger - lui ou certaines parties de lui-même; il est tombé dans une séduisante chausse-trape. Ainsi nous ne sommes pas seulement asservis, mais aussi pris au piège. L'artefact a délibérément projeté une illusion susceptible de nous mettre en transe et de nous attirer.

Toutefois, il arrive qu'un piège tel qu'une toile d'araignée (pour n'en citer qu'un parmi tant d'autres) attrape accidentellement une entité mortellement dangereuse, susceptible de tuer le constructeur du piège. Et c'est peutêtre le cas ici. Nous ne sommes peut-être pas ce que nous paraissons être, y compris à nos propres yeux.

Parfois, mais pas souvent, on peut faire remonter l'existence du mal à la nature duelle de Dieu lui-même. J'ai déjà évoqué ici la nature duelle de Shiva et du Christ - surtout celle de Shiva, lequel est souvent représenté en dieu de la mort. En voici deux exemples:

Jakob Böhme. Selon I'Encyclopedia Britannica, Böhme dit que Dieu se développe par étapes et que le monde n'est qu'un reflet de ce processus. Anticipant Hegel, il affirme aussi que cet autodéveloppement divin se produit par l'intermédiaire d'une dialectique permanente, une perpétuelle tension des contraires, et que ce sont les attributs négatifs de cette dialectique que les hommes perçoivent comme étant le mal dans le monde. Même si, dans l'ensemble, il accorde autant d'importance à l'absolu 
qu'au relatif, sa vision du monde comme simple reflet du divin (vision qui, apparemment, refuse aux êtres vivants ce même autodéveloppement) tend vers le panthéisme. (Article «Panthéisme et panenthéisme».)

Au cours de mes formidables révélations de 1974 et de l'anamnèse qui les ont accompagnées, j'ai perceptuellement observé la combinaison de Dieu et de la réalité et leur progression, stade évolutif après l'autre, au moyen d'une dialectique, mais je n'ai pas perçu ce que j'ai appelé "le contre-attaquant aveugle », c'est-à-dire le versant malfaisant considéré comme partie intégrante de Dieu. Toutefois, même si je percevais cette dialectique entre bien et mal, je ne pouvais rien affirmer quant à la source de ce mal. Toutefois [sic], j'ai bel et bien vu le versant bienfaisant en faire usage contre son gré, puisque, étant aveugle, le contre-attaquant malfaisant pouvait être employé à des fins bienfaisantes.

Hans Driesch (1867-1941). (Je cite encore l'Encyclopedia Britannica.) Driesch dit: [en termes métaphysiques] «Mon âme et mon entéléchie sont, dans la sphère de l'Absolu, une seule et même chose. » Et c'est seulement au niveau de l'Absolu qu'on peut parler d'« interaction psychophysique ». Mais l'Absolu ainsi compris transcende les limites possibles de nos connaissances, et ce serait commettre «une erreur que de prendre, à l'instar de Hegel, la somme de ses empreintes pour le Tout». L'étude de la vie mentale à l'état normal ne peut que nous conduire au seuil de l'inconscient; c'est dans la rêverie et dans certains cas anormaux de la vie mentale qu'on peut accéder aux «profondeurs de l'âme». II dit aussi plus loin que le fait d'éprouver le «sens du devoir » indique [l'existence d'un] Tout supra-personnel [en constante évolution] ainsi que la direction prise par ce développement supra-personnel. Mais le but ultime demeure inconnu. C'est là que l'Histoire se met à revêtir aux yeux de Driesch son sens particulier. D'un bout à l'autre, son œuvre se donne une orientation essentiellement empirique. Par conséquent, toute argumentation portant sur la nature du Réel ultime se doit d'être exclusivement hypothétique. On part du constat d'une «évidence » émergeant d'un «fond » conjectural. Le principe directeur de Driesch, en métaphysique, se ramène à ceci: le Réel que je postule doit être constitué de telle manière qu'il postule implicitement toutes nos expériences. Si l'on pouvait concevoir et postuler un tel Réel, alors toutes les lois de la nature, tous les principes et formules vrais des sciences fusionneraient avec lui, toutes nos expériences seraient «expliquées» par lui. De plus, puisque notre expérience est un mélange de totalité (le règne organique et le règne mental) et de non-totalité (le monde matériel), la Réalité elle-même doit être telle que je peux postuler un fondement dualiste de l'entièreté de mon expérience. En fait, pour jeter un pont - ah non, merde. Je reprends: En fait, il n'y a rien, pas même au sein de l'ultimement Réel, pour jeter un pont entre totalité et non-totalité. Ce qui signifie pour Driesch qu'en dernière analyse, il y a soit Dieu et «non-Dieu », soit un dualisme au sein de Dieu lui-même. Autrement dit, il peut y avoir soit un théisme de tradition judéo-chrétienne, soit un panthéisme centré sur un Dieu perpétuellement «en train de se faire " et transcendant ses propres stades antérieurs; les deux sont, en dernière analyse, compatibles avec les données de l'expérience. Driesch se trouvait dans l'impossibilité de choisir. Mais il était certain de ne pouvoir se satisfaire d'un monisme matérialiste/mécaniste. (Article «Driesch, Hans Adolf Eduard (1867-1941)».)

II semble que Böhme et $T$... je suis au bout du rouleau, là; je ne suis même plus capable de taper à la machine, alors pour ce qui est de réfléchir... Donc: que Böhme et Driesch parlent de la même chose, et que tous deux soient des philosophes (ou des théologiens, comme Whitehead) du process. Tous deux mettent en évidence la nature dialectique de Dieu. Driesch la voit à l'œuvre dans l'Histoire. II s'agit très certainement de la dialectique que j'ai vue pendant mes révélations de mars 1974 et, je le reconnais volontiers, il se peut très bien que le contreattaquant aveugle, malveillant, contre lequel œuvrait l'élément vitaliste bienveillant soit «Dieu en ses premiers stades », pour citer Driesch. Ce que j'apprécie chez lui, c'est que parvenu à un certain point, il ait simplement dit: «Je ne sais pas. " J'en suis là aussi, et ce n'est pas d'aujourd'hui; je ne sais tout simplement pas. Dieu a créé toute chose; le mal fait partie de ce grand tout; donc, Dieu est la source du mal - c'est ce que veut la logique, et dans le cadre du monothéisme, il n'y a pas d'échappatoire à cette argumentation. Si l'on postule deux dieux (ou plus), y compris un dieu mauvais, se pose la question de savoir d'où il vient. Mais le problème se pose aussi dans le monothéisme: s'il n'y a qu'un dieu, d'où vient-il? Réponse: il a la même origine que les divinités jumelles du dualisme. Autrement dit, cette question est aussi ardue dans le monothéisme que dans le dualisme. Nous ne savons tout simplement pas.

Cette hypothèse du mal comme succession de phases précoces chez un dieu en évolution, comme étape qu'il œuvre à dépasser, concorde très bien avec mes propres révélations, ma foi; elle est en syntonie avec moi. II m'a été montré comment tout ça fonctionne, mais je n'ai pas saisi ce que je voyais; c'est à une marionnette pas 
très futée qu'on le montrait - du genre Mortimer Snerd. J'ai bien eu le sentiment que j'assistais à une partie d'échecs cosmiques, le monde tenant lieu d'échiquier, et que l'un des deux joueurs (celui qui gagnait) était bienveillant tandis que l'autre n'était ni gagnant ni bienveillant; cet autre joueur était très puissant, mais handicapé par le fait qu'il était aveugle. Le bon côté, étant doté d'une sagesse infinie, pouvait prévoir l'avenir de manière absolue, donc préparer ses coups longtemps à l'avance; et le mauvais, le contre-attaquant aveugle et malveillant, n'était pas capable d'anticiper ce qu'ils lui rapporteraient. C'était une vision encourageante. Le Bien l'emportait à l'issue de chacun de ses stratagèmes et battait invariablement son malveillant adversaire. Que demander de plus à une Suprême Vision de la Réalité Totale et Absolue? Qu'ai-je besoin de savoir d'autre? Score: le mal - zéro; le bien l'infini. Je vais donc m'arrêter ici, satisfait. Le décompte final parle de lui-même.

\section{page 35}

1. Les citations bibliques empruntent à la traduction de Louis Segond,

1880 (NdT).

\section{page 36}

2. Trad. François-René de Chateaubriand (NdT). 